\title{
Transcranial alternating current stimulation increases risk-taking behavior in the Balloon Analog Risk Task
}

\author{
Tal Sela ${ }^{1}$, Adi Kilim ${ }^{2}$ and Michal Lavidor ${ }^{1,2,3 *}$ \\ ${ }^{1}$ Department of Psychology, Bar Ilan University, Ramat Gan, Israel \\ 2 The Gonda Multidisciplinary Brain Research Center, Bar Ilan University, Ramat Gan, Israel \\ ${ }^{3}$ Department of Psychology, University of Hull, Hull, UK
}

\section{Edited by:}

Eldad Yechiam, Technion Israel

Institute of Technology, Israel

\section{Reviewed by:}

Eldad Yechiam, Technion Israel Institute of Technology, Israel Itzhak Aharon, The Interdisciplinary Center, Israel

Giorgio Coricelli, University of Southern California, USA

*Correspondence:

Michal Lavidor, The Gonda Multidisciplinary Brain Research

Center, Bar Ilan University, Ramat Gan 52900, Israel.

e-mail:michal.lavidor@gmail.com
The process of evaluating risks and benefits involves a complex neural network that includes the dorsolateral prefrontal cortex (DLPFC). It has been proposed that in conflict and reward situations, theta-band $(4-8 \mathrm{~Hz})$ oscillatory activity in the frontal cortex may reflect an electrophysiological mechanism for coordinating neural networks monitoring behavior, as well as facilitating task-specific adaptive changes. The goal of the present study was to investigate the hypothesis that theta-band oscillatory balance between right and left frontal and prefrontal regions, with a predominance role to the right hemisphere $(\mathrm{RH})$, is crucial for regulatory control during decision-making under risk. In order to explore this hypothesis, we used transcranial alternating current stimulation, a novel technique that provides the opportunity to explore the functional role of neuronal oscillatory activities and to establish a causal link between specific oscillations and functional lateralization in risky decisionmaking situations. For this aim, healthy participants were randomly allocated to one of three stimulation groups (LH stimulation/RH stimulation/Sham stimulation), with active AC stimulation delivered in a frequency-dependent manner (at $6.5 \mathrm{~Hz} ; 1 \mathrm{~mA}$ peak-to-peak). During the AC stimulation, participants performed the Balloon Analog Risk Task. This experiment revealed that participants receiving LH stimulation displayed riskier decision-making style compared to sham and $\mathrm{RH}$ stimulation groups. However, there was no difference in decision-making behaviors between sham and $\mathrm{RH}$ stimulation groups. The current study extends the notion that DLPFC activity is critical for adaptive decision-making in the context of risk-taking and emphasis the role of theta-band oscillatory activity during risky decision-making situations.

Keywords: DLPFC, BART, risk-taking, theta-band oscillations, lateralization

\section{INTRODUCTION}

When facing risky situations humans have to weigh up the consequences of failure against the rewards for success. Assessing risk inevitably involves a conflict between the desire to win and the fear of penalty. In such situations, the ability to identify and weight risks and benefits is highly important in order to make proper predictions concerning potential outcomes that will best serve individual survival and future goals. In this regard, the cognitive architecture, neural, and electrophysiological basis of decision-making processes in the context of risk-taking has gained a lot of attention in the last two decades. Studies of patients with focal brain lesion (e.g., Bechara et al., 1994, 1996; Tranel et al., 2002), alongside numerous neuroimaging and electroencephalogram (EEG) studies (e.g., Rogers et al., 1999; Paulus et al., 2001; Sanfey et al., 2003a,b; Ernst and Paulus, 2005; Trepel et al., 2005; Krain et al., 2006; Rao et al., 2008; Gianotti et al., 2009; Hare et al., 2009; Mohr et al., 2010) suggest that decision-making processes involve a distributed subcortical-cortical network that includes multiple prefrontal, parietal, limbic, and subcortical regions.

Within this network, prefrontal cortex (PFC) involvement appears to be vital in decision-making under risk. Based on traumatic brain injuries or other pathologies affecting the PFC (Bechara et al., 1996; Rahman et al., 2001) it seems that PFC dysfunction typically manifests in a tendency for riskier decisionmaking behavior and an apparent disregard for negative consequences of actions during risky decision-making. In particular, the dorsolateral prefrontal cortex (DLPFC) has been considered to play an important role in decision-making under risk, probably due to its function in executive control, goal maintenance, and inhibitory control (Miller and Cohen, 2001; Knoch et al., 2006; Rao et al., 2008; Hare et al., 2009), as well as decision implementation (Mohr et al., 2010). This hypothesis seems particularly plausible for right hemisphere $(\mathrm{RH})$ role in risky decision-making under risk ("RH hypothesis"), and mostly pronounced in right PFC/DLPFC function as found in patients with right-sided lesions (Tranel et al., 2002; Clark et al., 2003), and is supported by several neuroimaging, EEG, and brain stimulation studies (e.g., van't Wout et al., 2005; Knoch et al., 2006; Fecteau et al., 2007a; Gianotti et al., 2009), and by a recent meta-analysis (Mohr et al., 2010). For instance, a repetitive transcranial magnetic stimulation (rTMS) study showed that individuals displayed riskier decision-making in a standard gambling paradigm after disruption of the right, but 
not the left, DLPFC (Knoch et al., 2006). Mohr et al. (2010) found that the right DLPFC (in conjunction with parietal cortex) has a role in risk processing during decision-making, particularly in the implementation of the risk decision, and the integration of the risk information with other aspects that may be relevant.

However, several findings call in to question the RH hypothesis role in risky decision-making under risk. For instance, a transcranial direct current stimulation (tDCS) study showed that after bilateral DC stimulation individuals displayed a conservative, riskaverse response style in a standard gambling paradigm (Fecteau et al., 2007b). In this study, unilateral DC stimulation to left or right DLPFC did not affect decision-making style at all, whereas both kinds of bilateral DC stimulations, regardless of electrodes polarity, produced the same behavioral outcome. Furthermore, in another tDCS study it has been found that DC modulation of the DLPFC influenced driving behavior, with anodal excitation of both the left and the right DLPFC leads to a more careful driving behavior (Beeli et al., 2008). Similar to Fecteau et al. (2007b), Beeli et al. (2008) did not find any clear functional lateralization patterns. These findings add to previous studies and suggestions such as Clark et al. (2003) report that patients with left-sided prefrontal lesions also displayed abnormal risk-taking behaviors, and to a meta-analysis of different neuroimaging studies which revealed that risky and ambiguous decision-making elicited activity bilaterally in the PFC (mainly orbitofrontal and DLPFC; Krain et al., 2006). This variety of evidence suggests that functional DLPFC lateralization in risk-taking behavior is still an unsolved issue that calls for further examination. Moreover, past studies, mostly studies that utilized brain stimulation techniques such as TMS and tDCS are restricted in the way they can uncover what is the electrophysiological mechanism that underlies the cognitive process in question.

Regional patterns of oscillatory activities can take place according to the behavioral tasks on which the brain is currently engaged (Thut and Miniussi, 2009). Studies into the role of brain oscillations in conflict and reward situations have demonstrated the relevance of oscillations in the theta-band $(4-8 \mathrm{~Hz})$. In particular, theta-band oscillatory activity over the medial frontal cortex has been proposed to reflect an electrophysiological mechanism for coordinating neural networks involved in monitoring behavior and the environment as well as facilitating task-specific adaptive changes in performance in conjunction with lateral PFC and sensory-motor areas. Different studies have identified that an induced oscillatory response in the theta-band during feedback processing is greater in power and phase coherence following negative feedback or errors relative to positive feedback or wins (e.g., Luu and Tucker, 2001; Luu et al., 2003, 2004; Cohen et al., 2007, 2008; Marco-Pallares et al., 2008; Cavanagh et al., 2009, 2010; Christie and Tata, 2009; van de Vijver et al., 2011). Furthermore, when an action or outcome is suboptimal and medial frontal cortex signals a need for adjustment, this also appears to lead to an increase in cognitive control, possibly via the additional recruitment of lateral PFC (Kerns et al., 2004; Ridderinkhof et al., 2004). Lateral PFC is assumed to adjust higher-level decisionmaking strategies to changing contexts and demands and to integrate information over time (McClure et al., 2004; Lee and Seo, 2007).
There is some evidence for the lateralization of the electrophysiological mechanism involved in risk-taking behavior. Gianotti et al. (2009) reported that individual's tonic cortical lateral PFC asymmetry in theta and delta bands predicted their behavior in a standard gambling paradigm. In other words, the extent to which baseline slow-wave oscillations in theta and delta bands was greater in the RH than in the left hemisphere, was positively associated with level of risk taken in Slovic's (1966) risk task. Specifically, using a source localization technique, they found that the baseline cortical activity in the right PFC predicts individual risk-taking behavior. A recent study by Christie and Tata (2009) showed that feedback-induced theta during the Iowa gambling task (IGT) was substantially right lateralized. Christie and Tata's (2009) finding adds to previous suggestions (Gehring and Willoughby, 2004; Marco-Pallares et al., 2008), which promote the hypothesis that medial frontal theta and the recruitment of right lateral PFC reward-related theta-band oscillatory activity may be regarded as the electrophysiological mechanism which mediates decision-making processes during risk-taking situations. In the current study, we aim to investigate this hypothesis and specifically the notion that theta-band oscillatory balance between right and left regions, with a predominance role to the $\mathrm{RH}$, is crucial for regulatory control during decision-making under risk. To the best of our knowledge, no past study has reported a direct causal link between oscillations and lateralization patterns to risky decisionmaking behaviors. In order to investigate this hypothesis, we used a novel stimulation technique called transcranial alternating current stimulation (tACS).

Transcranial alternating current stimulation provides a powerful approach to establish the functional role of neuronal oscillatory activities in the human brain and to explore the functional role of neural oscillations in cognitive tasks by stimulating the brain with biophysically relevant frequencies during task performance. tACS is supposed to induce regional brain oscillations in a frequencydependent manner, thereby interacting with specific functions of the stimulated region (Kanai et al., 2008, 2010; Pogosyan et al., 2009; Thut and Miniussi, 2009; Zaehle et al., 2010; Paulus, 2011). This technique is still largely unexplored and volume conduction effects are not wholly understood (Kanai et al., 2010; Zaghi et al., 2010; Feurra et al., 2011; Schutter and Hortensius, 2011). Nevertheless, recent studies have demonstrated tACS efficiency in different domains. For instance, Kanai et al. (2010) showed that cortical excitability of the visual cortex as measured by the thresholds for TMS evoked phosphenes, exhibits frequency dependency whereby $20 \mathrm{~Hz}$ tACS over the visual cortex enhances the sensitivity of the visual cortex. A recent study by Zaehle et al. (2010) provided direct physiological evidence of interaction between tACS and ongoing alpha oscillation in the occipital region. When tACS was delivered at the alpha-frequency, entrainment of the EEG amplitude in this frequency was observed. A recent study demonstrated that stimulation in alpha and gamma bands over the associative sensory cortex induced positive sensory sensations (Feurra et al., 2011). It has also been demonstrated that tACS at prefrontal sites during sleep improved procedural memory consolidation (Marshall et al., 2006).

Transcranial alternating current stimulation differ from other stimulation techniques that modulate brain frequencies, most 
notably rTMS. In general, low-frequency rTMS $(<1 \mathrm{~Hz})$ is often used to decrease excitability in an off-line mode (e.g., the task is administrated after the stimulation). In contrast, AC stimulation can possibly lead to one of two outputs: by inducing synchronous changes in brain activity, the AC stimulation can enhance ongoing oscillations and to increase/enhance cortical excitability, or AC stimulation can interrupt with ongoing cortical activity by introducing cortical noise, thus disrupt cortical excitability. This technique therefore allows us to exploit both properties of "enhancement" and "interference" in an on-line paradigm.

In the current study, we investigated whether on-line tACS can modulate the neural excitability of left and right PFC in a frequency-dependent manner. We aimed to examine whether risktaking strategies can be modified in healthy individuals and to provide direct evidence for the causal role of lateralized hemispheric control, frequency-dependent, of risk-taking during a gambling game. Specifically, we focused on the theta-band $(4-8 \mathrm{~Hz})$ as the main oscillatory frequency and the DLPFC as the main structure of interest. In the current experiment, participants were randomly allocated to one out of three stimulation conditions that included right or left AC stimulation, or a sham stimulation, and performed the Balloon Analog Risk Task (BART; Lejuez et al., 2002) during the AC stimulation.

The BART is a task which involves learning from experience (i.e., experience-based decision), that was originally developed to be used as a behavioral measure of risk-taking tendencies. The task has been found to have a convergent validity with real-world risk-related situations, and provides an ecologically valid model to assess human risk-taking propensity and behavior (Lejuez et al., 2002; Schonberg et al., 2011). The average number of adjusted pumps a person tolerates in the task was found to correlate with self-reported drinking, smoking, risky sexual behaviors, and substance use in healthy adults and adolescents (Lejuez et al., 2002, 2003a,b, 2004, 2005; Aklin et al., 2005; Hunt et al., 2005).

We predicted that AC stimulation over the right DLPFC would increase $\mathrm{RH}$ theta-band power; consequently, participants would display a more conservative, risk-averse response style (i.e., smaller number of average adjusted pumps during the BART compare to sham). On the other hand, AC stimulation over left DLPFC was predicted to increase LH theta-band power, thus violate the hemispherical balance, and to disrupt decision-making processing; thus, we expected that participants would display riskier decisionmaking style (i.e., larger number of average adjusted pumps during the BART compare to sham). Finally, we investigated whether individual differences such as gender and trait motivation characteristics may moderate tACS effectiveness on performance, since both factors have been suggested to moderate decision-making processes to some extent (Tranel et al., 2005; Demaree et al., 2008).

\section{MATERIALS AND METHODS PARTICIPANTS}

Participants in the experiment were 27 healthy college students (mean age $=23.89 \mathrm{SD}=2.45$; range $18-30$ years, 13 male, 14 female), each participant received 40 Shekel (equivalent to $\sim 10 \$$ ) for participating in the experiment. All participants gave informed consent in accordance with the Declaration of Helsinki and the procedures had the approval of the local ethics committee.
Participants had no metallic implants, previous history of any neurological disorders, medication, or substance abuse. All participants were right-handed as assessed by the Edinburgh Handedness Inventory (handedness score $\geq 90$; Oldfield, 1971). The participants were randomly allocated to one of three stimulation groups [LH stimulation $(N=9) / \mathrm{RH}$ stimulation $(N=8) /$ Sham stimulation $(N=10)]$.

\section{BALLOON ANALOG RISK TASK}

In the BART (Lejuez et al., 2002; Hunt et al., 2005), participants have to make a choice in a context of increasing risk. Participants inflated a computerized balloon by pushing a "pump" button. The balloon can explode at any moment. Participants have to decide after each pump whether to keep pumping and risk explosion, or to stop. In our modified version of the BART, participants accumulated points in a temporary bank with each pump (10 points). When the participant decided to stop pumping, the accumulated points transferred to a permanent bank. However, if the balloon explodes, all of the points accumulated in the temporary bank were lost. The probability that a balloon would explode was fixed at 1/128 for the first pump. If the balloon did not explode after the first pump, the probability that the balloon would explode was $1 / 127$ on the second pump, 1/126 on the third pump, and so on until the 128th pump the probability of an explosion was $1 / 1$ or a certainty. According to this algorithm, the average breakpoint was 64 pumps. Detailed instructions provided to the participants were based on those provided by Lejuez et al. (2002). Following instructions and a short guided practice, the task was administered until 30 balloons (i.e., trials) were completed. Note that participants did not actually receive the final sum of points stored in the permanent bank. Instead, they were informed at the beginning of the session that they are part of a tournament in which they play against other participants, for the prize of 250 Shekel (equivalent to $\sim 70 \$$ ), and their objective was to obtain the largest amount of points possible in order to win the prize.

Similar to previous studies that used the BART (e.g., Lejuez et al., 2002), the main outcome measure of the current examination was the adjusted number of pumps. In addition, total number of balloon explosions on the BART was calculated. Adjusted values were calculated based on the average number of balloon pumps on those balloons that did not explode. Adjusted values are preferable, because including balloon pumps from all trials (including those in which balloons exploded) result in the inclusion of trials in which the participants were forced to stop pumping because of the explosion (Lejuez et al., 2002; Aklin et al., 2005). Because the adjusted value consisted only of no-explosion trials, it considers being an index of a more adaptive (non-punitive) form of risk-taking behavior (Hunt et al., 2005). In contrast, evaluating the frequency of balloon explosions provided an index of a more maladaptive form of risk-taking whereby risk exceeded an acceptable level and ultimately was punished (via explosion and loss of money; Hunt et al., 2005). Furthermore, because the BART was performed during the whole stimulation duration, we calculated the time course of this measures (adjusted number of pumps and frequency of balloon explosions for three blocks, each block contain 10 balloons). 


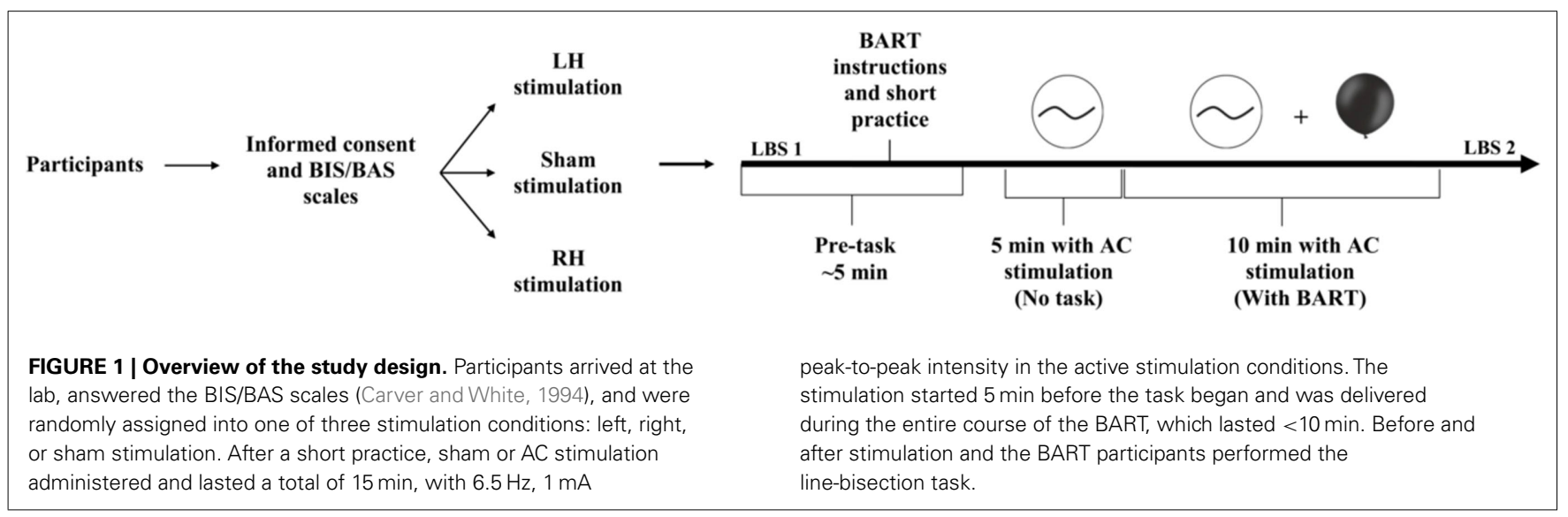

In addition, a recent advance in modeling methods of the BART task, originally introduced by Wallsten et al. (2005), validated recently by Bishara et al. (2009), and further developed by van Ravenzwaaij et al. (2011) propose a model where BART performance is governed by different component processes such as risk-taking (involving the tradeoff between reward and penalties) and general sensitivity to payoff which affects task performance. Whereas adjusted values and frequency of balloon explosions are usually considered to tap the construct of risk-taking, payoff sensitivity can be measured with the evaluation of participants' deviation from the optimal expected-value strategy. We report these measures in the Results section.

\section{tACS AND GENERAL PROCEDURE}

A double blind, randomized and sham-controlled trial was used in a between participants design (see Figure 1). The experiment included three types of stimulation, two active stimulation conditions and one sham condition. We used the international EEG 10/20 system to determine stimulation sites. To stimulate the $\mathrm{LH}$, one electrode was placed over the left DLPFC (F3) and the reference electrode was placed over the left temporal (CP5). To stimulate the RH, one electrode was placed over the right DLPFC (F4) and the reference electrode was placed over the right temporal (CP6). For sham stimulation, the electrodes were placed in the same positions as for active conditions (half of the participants with LH montage and the other half with $\mathrm{RH}$ montage).

The stimulation started 5 min before the task began and was delivered during the entire course of the BART, which lasted $<10 \mathrm{~min}$. tACS was induced by two $5 \mathrm{~cm} \times 5 \mathrm{~cm}$ saline-soaked synthetic sponge electrodes and delivered by a battery-driven, constant-current stimulator (Magstim Ltd., Wales). The waveform of the stimulation was sinusoidal and there was no DC offset. AC was delivered at a frequency of $6.5 \mathrm{~Hz}$ and the intensity was $1 \mathrm{~mA}$ (peak-to-peak). For active stimulation conditions the AC stimulation was delivered for $15 \mathrm{~min}$. For sham stimulation, stimulation was delivered for $30 \mathrm{~s}$ and then turned off. Thus, participants felt the initial itching sensation associated with brain stimulation but received no active current for the rest of the stimulation period. This method of sham stimulation has been shown to be reliable with respect to DC stimulation (Gandiga et al., 2006). In the present study participants were kept blinded with regard to the type of the stimulation; the AC procedure used, with AC delivered at a frequency of $6.5 \mathrm{~Hz}$, did not induce any flickering sensation or any other side effects, as verified by questioning participants after the stimulation.

\section{ASSESSMENT OF MOTIVATION}

At the start of the session, participants completed the BIS/BAS scales (Carver and White, 1994) in order to evaluate trait motivational characteristics. The BIS/BAS scales (Carver and White, 1994) measures two independent based dimensions of motivation (Gray, 1987; Pickering and Gray, 1999; Gray and McNaughton, 2000): the BAS, which regulates responses to rewarding stimuli, and the BIS, which regulates inhibitory processes to aversive stimuli. All items were judged on a four-point scale ranging from 1 ("I strongly agree") to 4 ("I strongly disagree"). The BIS/BAS scales assess one behavioral inhibition measure (BIS; e.g., "I worry about making mistakes") and three personality measures related behavioral approach (BAS): (1) The positive anticipation of rewarding events (BAS Reward Responsiveness - BAS RR; e.g." "When I see an opportunity for something I like I get excited right away"); (2) Items tapping strong pursuit rewards (BAS Drive - BAS D; e.g., "I go out of my way to get things I want"); (3) The tendency to seek out new rewarding situations (BAS Fun Seeking - BAS F; e.g., "I am always willing to try something new if I think it will be fun").

\section{LINE-BISECTION}

Before and immediately after BART performance and AC stimulation, participants performed two line-bisection trials as a simple and non-invasive behavioral measure of a hemispheric bias. On each trial, participants were asked to mark the exact center of a 180 -mm black line printed horizontally on a white sheet of paper. The line was printed at mid height of the page and was closer to the right border on one trial and closer to the left border on the other. Participants used a fine-point pen to bisect the line as accurately as they could. Scores reflected the percent of deviation from the center of the line: positive scores reflect a bias to the right side (stronger LH activation), and negative scores reflect a bias to the left side (stronger RH activation; Goldstein et al., 2010; Nash et al., 2010). 


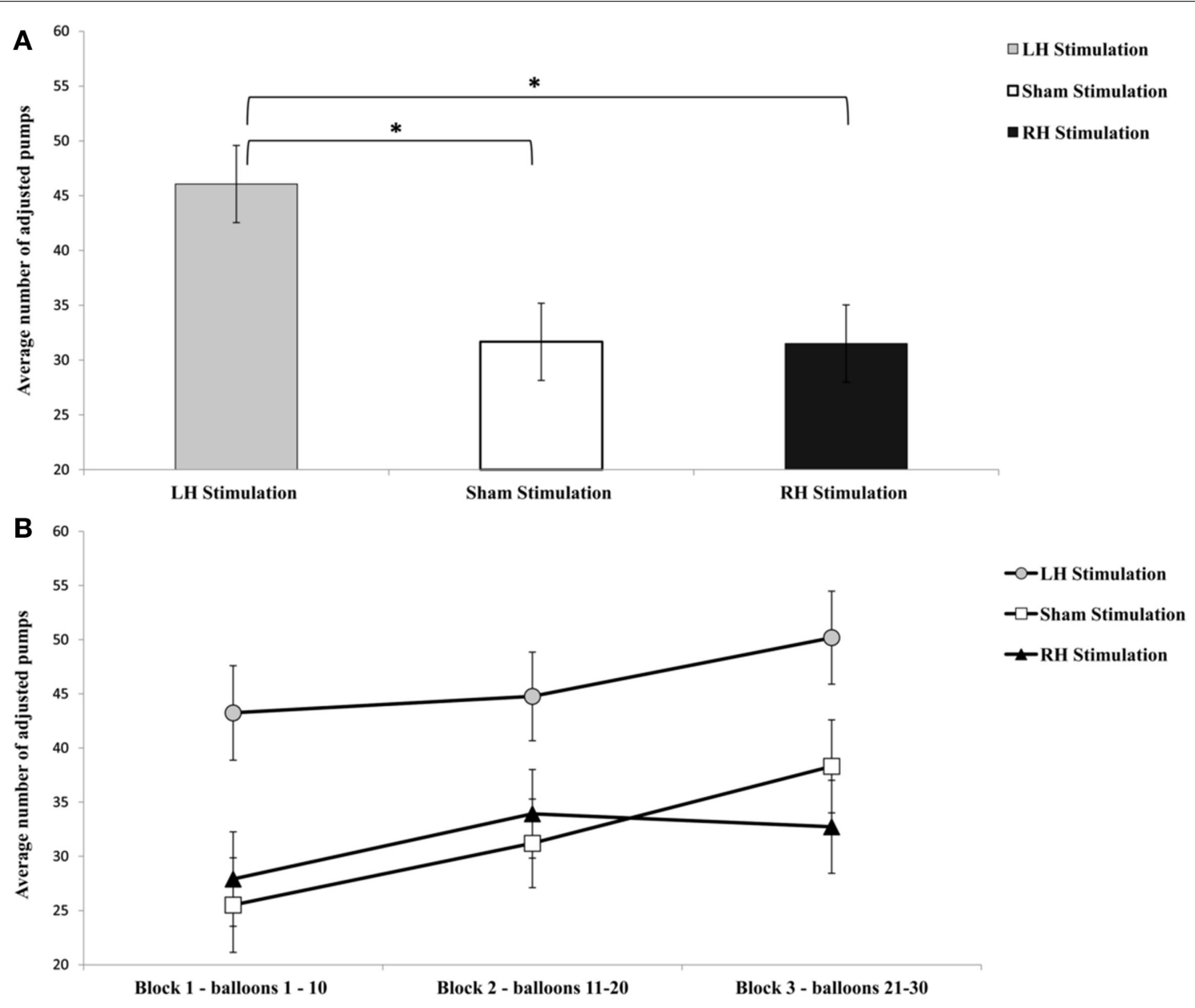

FIGURE 2 | Graphic display of the average number of adjusted pumps (the total pumps of the balloon that did not explode) for each stimulation group (A) and the average number of adjusted pumps for each group and time period (B). Error bars indicate SEM. ${ }^{*} p<0.05$.

\section{RESULTS}

\section{BART PERFORMANCE}

The data from the BART task was analyzed with a mixed AVOVA model that included one between-subject factor and one within-subject factors. The between-subject factor was Stimulation Group (LH stimulation/RH stimulation/sham stimulation) and the within-subject factor was Time (first block/second block/third block). Average number of adjusted pumps and total number of balloon explosions served as the dependent variables. When relevant, post hoc analyses were performed using a Bonferroni correction for multiple comparisons. Three participants (two in the sham group and one in the LH stimulation group) were excluded from all analyses as outliers (2 SD above or below the mean of the group for the adjusted number of pumps).

The analysis of average number of adjusted pumps revealed a main effect for Stimulation Group $\left[F_{(2,21)}=5.63, p<0.05\right.$; $\eta_{\mathrm{p}}^{2}=0.35$; see Figure 2]. Post hoc tests revealed an effect and show that the LH stimulation group differed significantly from both the sham stimulation $(p<0.05)$ and $\mathrm{RH}$ stimulation $(p<0.05)$ groups. In addition, the analysis revealed a main effect for Time $\left[F_{(2,42)}=5.93, \mathrm{p}<0.05 ; \eta_{\mathrm{p}}^{2}=0.22\right]$. A trend analysis showed a linear trend across blocks one to three $\left[F_{(1,21)}=7.60, p<0.05\right.$; $\left.\eta_{\mathrm{p}}^{2}=0.26\right]$. Post hoc tests reinforced this linear trend, and revealed that the first and the last blocks differed significantly $(p<0.05)$. However, the analysis did not reveal any significant interaction between the two factors $(F<1)$.

The analysis of total number of balloon explosions also revealed a main effect for Stimulation Group $\left[F_{(2,21)}=6.63, p<0.01\right.$; $\eta_{\mathrm{p}}^{2}=0.39$; see Figure 3A]. Post hoc tests revealed that the LH stimulation group differed significantly from sham stimulation group $(p<0.01)$, and marginally differed from RH stimulation group $(p=0.056)$. In addition, the analysis revealed a marginal effect for Time $\left[F_{(2,42)}=2.96, p=0.06 ; \eta_{p}^{2}=0.12\right]$. Post hoc tests revealed that there was no significance different between the first block $(M=3 ; \mathrm{SD}=1.56)$ and the second block $(M=2.75 ; \mathrm{SD}=1.32)$. However, the second and the third block $(M=3.54 ; \mathrm{SD}=1.31)$ differed significantly $(p<0.001)$. The analysis did not reveal any significant interaction between the two factors $(F<1)$.

We further analyzed balloon explosions frequencies by defining for each participant whether a balloon explosion was a one-time explosion or a sequential explosion (a one-time explosion was defined as the number of total balloon explosions minus number 


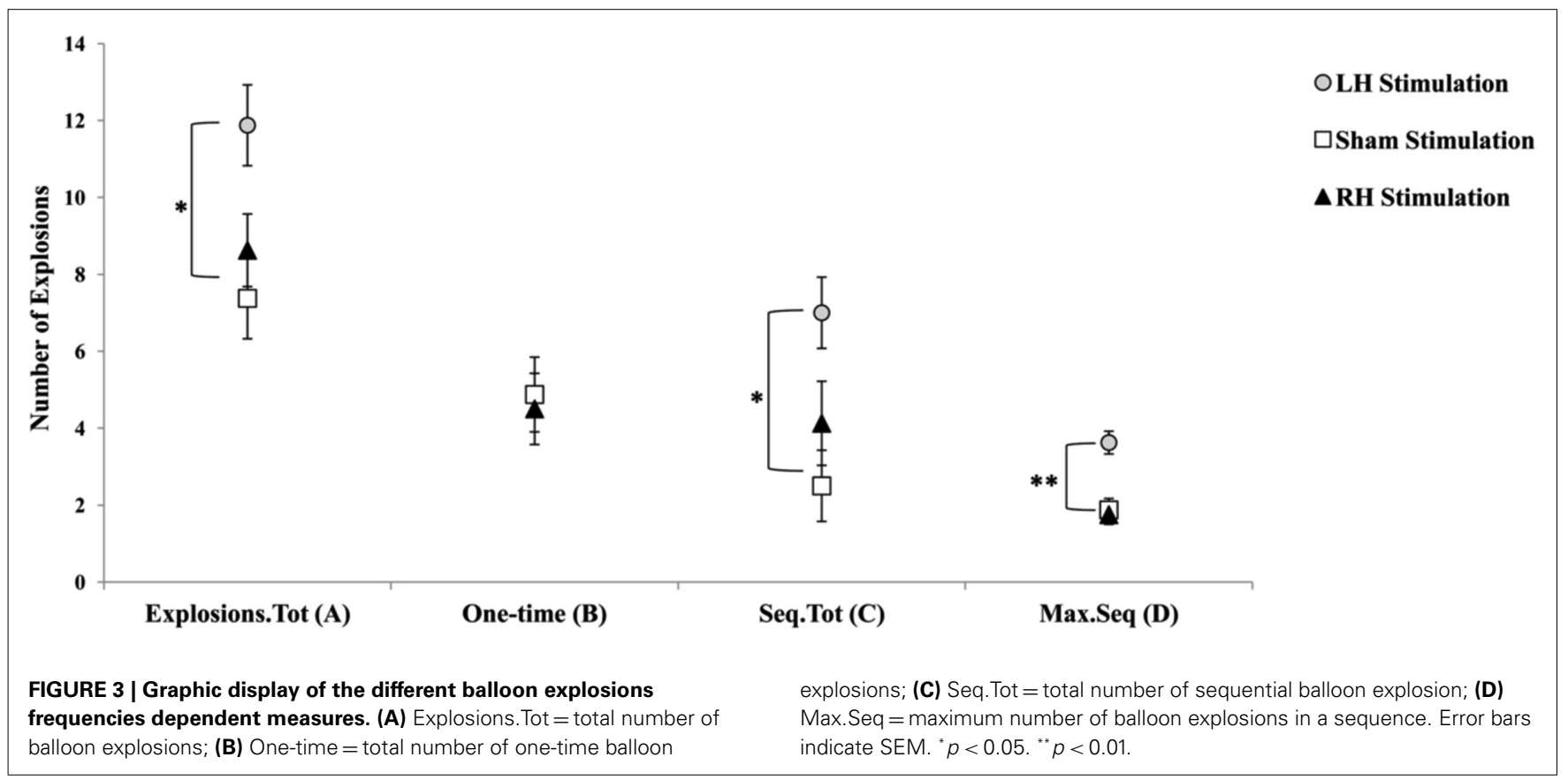

Table 1 | Pearson's correlations among different BART performance parameters.

(1)

$-$

(1) One-time

(2) Seq.Tot

(3) Max.Seq

(4) Pumps.Adj

(5) Points. Tot

(6) Explosions.Tot
(2)

-
$0.76^{* * *}$
$0.56^{* * *}$
$0.35^{*}$
$0.74 * * *$

(3)

$$
-0.22
$$

0.28

$0.35^{*}$

0.22

-
$0.65 * * *$
$0.47 * *$
$0.69 * * *$

(4)
(5)

One-time = total number of one-time balloon explosions; Seq. Tot=total number of sequential balloon explosion; Max.Seq= maximum number of balloon explosions in a sequence; Pumps.Adj= average adjusted number of pumps; Points. Tot = total points earned; Explosions. Tot $=$ total number of balloon explosions.

${ }^{*}$ Correlation is significant at the 0.05 level (one-tailed).

${ }^{* *}$ Correlation is significant at the 0.05 level (two-tailed).

${ }^{* * *}$ Correlation is significant at the 0.01 level (two-tailed).

of explosions in trial $n$ that were followed by no-explosion in trial $n+1$; sequential explosion was defined as the number of total balloon explosions minus total number of explosions in trial $n$ that were followed by an explosion in trial $n+1, n+2$, etc.; the two measures are complementary). In addition, based on this simple calculation, we also defined another variable term "Maximum sequential explosions" that reflected the highest number of balloon explosions in a sequence for each participant. Consequently, each participant had three additional measures of the original balloon explosions frequency. The rationale to use these indices is based upon the idea that the use of a maladaptive index of risk-taking (e.g., number of balloon explosions) in a task with a random schedule of explosions may create an artifact with respect to the actual number of explosions that were a result of a risk behavior that exceeded an acceptable level and resulted in an explosion. Overall, the new indices were used in order to verify whether participants in the LH stimulation group indeed tend to pump the balloon more, a tendency that may be manifested not only in a higher overall number of explosions compare to the two other stimulation groups, but particularly in a higher number of non-random explosions. Pearson's correlations coefficients between the three newly defined measures and the other original BART parameters were calculated and presented in Table 1. The correlations of number of sequential explosions and maximum sequential explosions to the other BART known parameters suggest that these variables are reliably correlated, contrary to the number of one-time explosions.

Furthermore, based on these new measures, we conducted a MANOVA with Stimulation Group as between-subject factor and each of the newly defined measures as the dependent variables. We reveled a Stimulation Group effect [Wilks' Lambda $=0.45$, $\left.F_{(6,38)}=3.13, p<0.05 ; \eta_{\mathrm{p}}^{2}=0.32\right]$. Follow-up testing showed that no stimulation group effect was found with respect to number of one-time explosion $(F<1$; see Figure 3B). However, a Stimulation 
Table 2 | Demographics and motivation information for the Sham, LH, and RH stimulation groups.

Variables and measures

\section{Group}

Sham stimulation $(N=8)$

LH stimulation $(N=8)$

RH stimulation $(N=8)$

\section{DEMOGRAPHICS}

Gender

Age

Years of education

\section{BIS/BAS SCORES}

BIS

BAS

BAS D

BAS FS

BAS RR

5 Males/3 females
25 (3.59)

$14.75(1.08)$
3 Males/5 females

22.87 (1.55)

$13.25(0.88)$
4 Males/4 females

23.62 (2.07)

14 (1.07)
$13.50(2.26)$

$22.75(6.92)$

7.87 (2.23)

7.37 (3.45)

$7.50(2.45)$
13.12 (2.53)

21.62 (4.68)

7.12 (2.85)

7.75 (1.90)

6.75 (1.83)
14.50 (3.20)

23.87 (5.44)

8.25 (1.91)

7.25 (2.12)

8.37 (2.13)

Values shown as mean (SD). BIS, behavioral inhibition system; BAS, behavioral approach system; BAS D, behavioral approach drive; BAS FS, behavioral approach fun seeking; BAR RR, behavioral approach reward responsiveness.

Group effect was found with respect to number of sequential explosions $\left[F_{(2,21)}=4.34, p<0.05 ; \eta_{\mathrm{p}}^{2}=0.29\right.$; see Figure $\left.3 \mathrm{C}\right]$. Post hoc tests revealed that the LH stimulation group differed significantly from the sham stimulation group $(p<0.05)$. A similar effect was reveled with respect to the maximum of sequential explosions $\left[F_{(2,21)}=7.61, p<0.01 ; \eta_{\mathrm{p}}^{2}=0.42\right.$; see Figure $\left.3 \mathrm{D}\right]$. Post hoc tests revealed robust effect and show that the LH stimulation group differed significantly from both the sham stimulation $(p<0.01)$ and RH stimulation $(p<0.01)$ groups. These analyses confirmed and elaborated on our previous mentioned results by demonstrating that participants who received the LH stimulation demonstrated a strategy of risky decision all along the BART, which systematically differed to the sham and RH stimulation groups. All the groups tolerated a similar number of one-time explosions, that resulted from the inherent nature of the task, but only participants receiving $\mathrm{LH}$ stimulation displayed a tolerance for losses, and in particular, sequential losses.

Lastly, we computed a behavioral index that taps participants' behavior in relation to optimal behavior in the BART task (e.g., payoff sensitivity). The optimal expected-value strategy was to pump 64 times and then stop. Explosion points were determined for each balloon in the manner described (i.e., each pump had an a priori probability of 1/128 of yielding an explosion) but with the constraint that explosions were scheduled to occur on average on Pump 64 over the entire 30 balloons and within each sub-block of 10 . We calculated the mean squared distance (MSD) of each participant number of pumps at a given trial from the optimal number of pumps. MSD therefore reflects participants' sensitivity to payoffs, so that a closer score to zero represents an optimal strategy. Pearson's correlations coefficients between this measure and the two main BART outcome parameters reported earlier (e.g., average number of adjusted pumps and total number of balloon explosions) were performed, and showed a very high correlation $(r=-0.91, p<0.000$; $r=-0.80, p<0.000$; for adjusted pumps and balloon explosions, respectively). The fact that these parameters are highly correlated indicates that payoff sensitivity and risk-taking measures are confounded.

\section{GENDER AND MOTIVATION BIAS}

We investigated a possible moderation effect of individual differences such as gender and trait motivation characteristics (see Table 2 for descriptive statistics) on performance. First, we separately entered gender as a covariate to the mixed AVOVA models reported earlier. There was no significant effect to gender or any significant interactions with other factors in any of the models. Second, in order to investigate the role of motivation bias on performance, we separately entered BAS, BIS, and BAS subscales scores as covariates to the mixed AVOVA models reported earlier. All models produced non-significant effects for motivation bias. The stimulation groups did not differ in any demographic variables or in any BIS/BAS parameter $(F<1)$.

\section{LINE-BISECTION BIAS}

In order to evaluate how BART performance and AC stimulation affected hemispheric bias as measured by the line-bisection, we analyzed line-bisection scores in a mixed ANOVA model that included Stimulation Group (LH stimulation/RH stimulation/sham stimulation) as the between-subject factor and Time (Before/After) as the within-subject factor. The analysis revealed a main effect for Time $\left[F_{(1,18)}=23.70, p<0.000 ; \eta_{\mathrm{p}}^{2}=0.53\right]$. The line-bisection index was more negative after performing the BART task $(M=-0.16, \mathrm{SE}=0.06)$ compare to before $(M=0.18$, $\mathrm{SE}=0.07)$, indicating that the BART had the expected hemispheric effect, i.e., RH engagement which lead to stronger RH activation. This asymmetry shift can be further emphasized - 18 out of 24 participants achieved a positive line-bisection score before the BART and $\mathrm{AC}$ manipulation (this was significantly higher than $50 \%$ by a binomial test, $p<0.05$ ), but after task and stimulation, 18 out of 24 achieved a negative score (this was significantly higher than $50 \%$ by a binomial test, $p<0.05$ ). We separately analyzed before and after line-bisection scores for the different AC groups using paired samples $t$-tests. Participants in the Sham $\left[t_{(7)}=3.25, p<0.05\right]$ and RH stimulation $\left[t_{(7)}=5.6, p<0.001\right]$ groups showed the asymmetry shift, however participants in the LH stimulation showed only a non-significant trend $\left[t_{(7)}=1.47, \mathrm{n} . \mathrm{s}\right]$. This finding implies 
that the BART did not produce the expected asymmetry shift within the LH stimulation group.

\section{DISCUSSION}

The current study explored the cognitive architecture, neural, and electrophysiological basis of decision-making processes in the context of risk-taking. Overall, we report that participants receiving AC stimulation of $6.5 \mathrm{~Hz}$ to the $\mathrm{LH}$, with one electrode located over the left DLPFC and the reference electrode located over left temporal cortex, displayed a risky response style, making more pumps on the BART, and tolerated a larger number of balloon explosions than those with sham stimulation and those with $\mathrm{RH}$ stimulation. This is the first study showing that neuromodulation in the theta-band can causally modulate decision-making style in healthy participants. In addition, this result supports previous evidence showing that the DLPFC is causally involved in modulating risky decision-making behaviors.

The current result supports, to some extent, the hypothesis that the theta-band oscillatory balance between right and left regions is crucial for regulatory control during decision-making under risk. As predicted, participants receiving AC stimulation to the $\mathrm{LH}$ displayed a risky response style. It has been proposed that in conflict and reward situations, theta-band oscillatory activity over the frontal medial cortex may reflect an electrophysiological mechanism for coordinating neural networks involved in monitoring behavior and the environment as well as facilitating task-specific adaptive changes. Furthermore, induced oscillatory response during feedback processing found to be greater in power and phase coherence following negative feedback or errors relative to positive feedback or wins (Luu and Tucker, 2001; Luu et al., 2003, 2004; Cohen et al., 2007, 2008; Marco-Pallares et al., 2008; Cavanagh et al., 2009, 2010; Christie and Tata, 2009; van de Vijver et al., 2011). We propose that AC stimulation at the theta-band to the LH, created continuous disruption to participants' ability to process and adjust their actions based on negative feedback or errors, as shown by their persistent tendency to tolerate losses, and in particular, sequential losses. We further claim that the balance between right and left regions, and in particular, the predominance of the RH, is needed in order to be able to adopt a conservative, risk-averse response style during the BART. Since we interfered with this balance and especially with RH dominance, participants lacked the ability to adjust their risk-taking behaviors and tend to display a risky response style.

Previous studies addressed the relative contribution of the right and the left prefrontal regions in risk-taking behaviors and particularly the role of the DLPFC in this kind of behavior. Various studies have provided clear evidence for the role of the right DLPFC in decision-making and risk-taking situations. Using low-frequency rTMS van't Wout et al. (2005) found a disruption to the right DLPFC resulted in accepting more frequently unfair offers and taking longer to refuse unfair offers. Knoch et al. (2006) reported that suppression of activity in the right but not the left DLPFC with low-frequency rTMS made participants choose high-risk prospects more often. Moreover, using a different brain stimulation methodology, i.e., tDCS, Fecteau et al. (2007b) showed that during right anodal/left cathodal stimulation over the DLPFC, participants chose more often the safe prospect compared with the sham and reversed polarization groups. However, other studies have not found clear lateralization effects (e.g., Fecteau et al., 2007b; Beeli et al., 2008). It has been suggested that divergent results from different brain stimulation studies might be due to differences in the risk-taking paradigm used and/or the method of stimulation involved (Fecteau et al., 2007b).

Our results are in line with the RH hypothesis in risk-taking behaviors, and address lateralization in terms of electrophysiological balance between left and right cortical regions in the theta bend. Previous suggestions (Gehring and Willoughby, 2004; Marco-Pallares et al., 2008; Christie and Tata, 2009) have already raised the hypothesis that right medial frontal/prefrontal theta may be regarded as the electrophysiological mechanism which mediates decision-making processes during risk-taking situations, and the present study adds a causal link between the electrophysiological mechanism and theta-band activity to actual behavior.

In addition, it is important to note that we address lateralization in terms of hemispheric shift. It has been recently reported that BART performance elicited greater activity in the right DLPFC (Rao et al., 2008) providing further support to previous studies of patients with right-sided lesions (Tranel et al., 2002; Clark et al., 2003) that reported on a dysfunction in risky decisionmaking behaviors. Apart from the main findings reported earlier, the simple asymmetry index (i.e., the line-bisection task) provided further support for this hypothesis, and showed that only in the sham and RH stimulation groups, but not LH stimulation group, line-bisection bias was more negative after performing the BART compare to baseline performance. This finding indicates that the BART had the expected hemispheric effect, i.e., a RH enduring engagement, which was reflected by a stronger RH activation in those groups only. Tendencies toward rightward versus leftward errors in estimating the actual midpoints are taken to reflect relative primacy of right versus left visual fields, respectively, and neural activity in the contralateral hemisphere (Kinsbourne, 1970; Milner et al., 1992; Goldstein et al., 2010). Even though previous research suggests line-bisection bias may be more a marker of parietal than prefrontal function (Vallar and Perani, 1986), the simple and non-invasive line-bisection task has been recently found to serve as a neural index of asymmetrical activity related to the DLPFC (Nash et al., 2010).

In the present study, we failed to find an effect for AC stimulation over the RH. We expected that following RH stimulation, participants would display a more conservative, risk-averse response style. The results suggest that participants who received this stimulation behave as participants in the sham stimulation. This null result can be marked as a "floor effect" and can be explained in terms of behavioral, methodological, and electrophysiological aspects. First, with respect to behavior, this "floor effect" probably represents a possible limitation of our ability to modulate risk-taking behavior in healthy participants, and to increase their risk-averse response style. It is possible that RH stimulation will be more affective with populations that show deficits in risk-taking tasks such as patients with lesions in the PFC and other clinical populations, such as drug abusers, alcoholics, and pathological gamblers (Bechara et al., 1996; Rahman et al., 2001). Second, this "floor effect" may be also a direct outcome of the task properties, in which it is easy to demonstrate what is considered to be a 
risky behavior (e.g., a large number of adjusted pumps and a large number of balloon explosions), but it may be harder to reveal an overcautious, conservative, risk-averse response style. Third, this "floor effect" can be referred as close to the idea of the so called "natural frequency" (Rosanova et al., 2009), by which different corticothalamic brain modules are tuned to oscillate at a topographically organized "natural frequency." It is possible that the AC stimulation to the RH interacted with the neuronal oscillatory activity that already evoked by the task, i.e., the "natural frequency" that characterizes the decision-making processes that usually take place during processing, thus did not modulate any cortical activity or risk-taking behavior.

Overall, our results suggest that during risk-taking situations, the hemispheric balance is important. This suggestion may account for previous conflicting results, mentioned earlier, regarding the relative contribution of the right and left PFC/DLPFC in risky decision-making behaviors. This balance can metaphorically be described as a seesaw between left and right frontal/prefrontal areas that is theta dependent. The right hemispheric shift is vital, and especially the recruitment of right lateral PFC, in order to promote a conservative, risk-averse response style. Hence, it is clear that right prefrontal regions must be functionally and anatomically intact in order to facilitate such an on-line shift. However, the LH is also crucial for this shift, and especially the balance between the two. Theta-band tonic activity balance between left and right prefrontal regions has been found to predict risk-taking behavior (Gianotti et al., 2009), showing the importance of the hemispheric balance right from a pre-stage of risk-taking situations. In addition, this hypothesis is similar to a novel framework of risk processing suggested by Mohr et al. (2010). Based on a meta-analysis on the neural basis of risky behavior, the authors proposed a potential mechanism of risky decision-making that involves two parallel and reciprocal risk processes; one is emotional and the other one is cognitive. These processes involve the anterior insula and the thalamus as the key regions which mediate emotional processing, whereas the dorsomedial PFC evaluates the risk of the stimulus on a cognitive level. According to their framework, both parts of risk processing (emotional and cognitive) inform the actual decision process performed in DLPFC and parietal cortex. It is possible that our hypothesis represents, to some extent, Mohr et al.'s (2010) framework, with the frontal/prefrontal hemispheric balance as the cognitive level of processing, and the mandatory recruitment of the right DLPFC as the exaction phase. This suggestion is reasonable given the findings that when the medial frontal cortex signals a need for adjustment, this also involves an additional recruitment of lateral PFC (Kerns et al., 2004; Ridderinkhof et al., 2004).

A final note, apart from matters of lateralization, the current study addressed the issue of cognitive processes that govern BART performance. Previous work highlighted the role of two key concepts, namely, risk-taking and payoff sensitivity (e.g., Bishara et al., 2009). However, in the current study, risk-taking and payoff sensitivity measures were highly correlated, and presumably are confounded, and means that in practice these definitions of performance are interchangeable, at least for the specific task paradigm used. Therefore, it is difficult to distinguish between these two-component processes and as a consequence to draw a firm conclusion to whether participants in the different stimulation groups were more risk-averse or risk seeking, since participants respond in a risk-aversive manner in general. This issue has been acknowledged previously (e.g., Freeman and Muraven, 2010). In the current experiment, average number of pumps per group was below the average explosion point across balloons (64, which is also the optimal number of pumps to maximize earnings), hence the group that pumped the balloon more earned more points. This finding is not unique to our experiment, as participants generally respond in a risk-aversive manner on the BART (see also Lejuez et al., 2002, 2003a; Bornovalova et al., 2009; Freeman and Muraven, 2010). Apparently, human subjects and also rats (see Jentsch et al., 2010) exhibit risk-averse profiles when performing the BART (or BART alike task in the case of rats), producing fewer than the optimal number of responses, and earning less than possible probably because of over-estimation of the risk associated with the task (Bornovalova et al., 2009; Jentsch et al., 2010).

Several limitations must be considered when interpreting the results. First, the present study used only one band of stimulation frequency, was restricted to specific locations, and measured behavioral effects of a particular risk-taking paradigm. Future research should elaborate the scope of reference and examine more bands, in various cortical locations using a verity of risktaking paradigms. Second, no direct assessment of DLPFC activity was made by any imaging technique before and/or after tACS stimulation, so any attempt to bond between DLPFC activity, tACS effects, and risk-taking behaviors call for further examination. Future research should document neural baseline and changes accruing after AC stimulation in order to be able to infer about the neural circuitry and the mechanisms that are influenced by AC stimulation. Third, we stimulated all participants in the active conditions in our study with $6.5 \mathrm{~Hz}$, thereby ignored possible inter individual variability that may be captured and elaborate our knowledge regarding the electrophysiological mechanism in question. For example, it is possible to stimulate each participant with her/his transition frequency (TF). TF shows a large inter individual variability ranging from about 4 to $7 \mathrm{~Hz}$ (Klimesch et al., 1996; Klimesch, 1999), so TF can be measured in order to create a tailored stimulation for each participant in future studies. Forth, in the present study we did not find that individual differences such as gender and/or trait motivation characteristics moderate tACS effectiveness on performance. Although we did not find a gender or motivation difference in the BART measures, additional studies should specifically explore whether there is a gender or motivational bias in decision-making in regards to brain stimulation. Finally, in the current study, we employed a procedure similar to the one used by Hunt et al. (2005), where participants did not actually receive money for their BART performance, rather they were competing for a monetary prize. It is possible that this kind of incentive procedure may have generated a competitive environment and may have bias choice behavior. Future research is needed to clarify this issue.

\section{CONCLUSION}

The current study report a novel finding demonstrating that neuromodulation in the theta bend can causally modulate 
decision-making style by increasing risk-taking behavior in healthy participants and provides further support to previous evidence by showing that the DLPFC is causally involved in modulating decision-making. This study may inspire the use of tACS to further examination of risky decision-making behaviors, and hopefully in the near future would be beneficial as a therapeutic tool for patients with different brain lesions and other clinical populations, such as drug abusers, alcoholics,

\section{REFERENCES}

Aklin, W. M., Lejuez, C. W., Zvolensky, M. J., Kahler, C. W., and Gwadz, M. (2005). Evaluation of behavioral measures of risk taking propensity with inner city adolescents. Behav. Res. Ther. 43, 215-228.

Bechara, A., Damasio, A. R., Damasio, H., and Anderson, S. W. (1994). Insensitivity to future consequences following damage to human prefrontal cortex. Cognition 50, 7-15.

Bechara, A., Tranel, D., Damasio, H., and Damasio, A. R. (1996). Failure to respond autonomically to anticipated future outcomes following damage to prefrontal cortex. Cereb. Cortex 6, 215-225.

Beeli, G., Koeneke, S., Gasser, K., and Jancke, L. (2008). Brain stimulation modulates driving behavior. Behav. Brain Funct. 4, 34.

Bishara, A. J., Pleskac, T. J., Fridberg, D. J., Yechiam, E., Lucas, J., Busemeyer, J. R., Finn, P. R., and Stout, J. C. (2009). Similar processes despite divergent behavior in two commonly used measures of risky decision making. J. Behav. Decis. Mak. 22, 435-454

Bornovalova, M. A., Cashman-Rolls, A., O'Donnell, J. M., Ettinger, K., Richards, J. B., Dewit, H., and Lejuez, C. W. (2009). Risk taking differences on a behavioral task as a function of potential reward/loss magnitude and individual differences in impulsivity and sensation seeking. Pharmacol. Biochem. Behav. 93, 258-262.

Carver, C. S., and White, T. L. (1994). Behavioral inhibition, behavioral activation, and affective responses to impending reward and punishment: the BIS/BAS scales. J. Pers. Soc. Psychol. 67, 319-319.

Cavanagh, J. F., Cohen, M. X., and Allen, J. J. B. (2009). Prelude to and resolution of an error: EEG phase synchrony reveals cognitive control dynamics during action monitoring. J. Neurosci. 29, 98-105.

Cavanagh, J. F., Frank, M. J., Klein, T. J., and Allen, J. J. B. (2010). Frontal theta links prediction errors to behavioral adaptation in reinforcement learning. Neuroimage 49, 3198-3209.
Christie, G. J., and Tata, M. S. (2009). Right frontal cortex generates reward-related theta-band oscillatory activity. Neuroimage 48 , 415-422.

Clark, L., Manes, F., Antoun, N. Sahakian, B. J., and Robbins, T. W. (2003). The contributions of lesion laterality and lesion volume to decision-making impairment following frontal lobe damage. Neuropsychologia 41, 1474-1483.

Cohen, M. X., Elger, C. E., and Ranganath, C. (2007). Reward expectation modulates feedback-related negativity and EEG spectra. Neuroimage $35,968-978$.

Cohen, M. X., Ridderinkhof, K. R., Haupt, S., Elger, C. E., and Fell, J. (2008). Medial frontal cortex and response conflict: evidence from human intracranial EEG and medial frontal cortex lesion. Brain Res. 1238, 127-142. K. J., and Erik Everhart, D. (2008). You bet: how personality differences affect risk-taking preferences. Pers. Individ. Dif. 44, 1484-1494.

Ernst, M., and Paulus, M. P. (2005). Neurobiology of decision making: a selective review from a neurocognitive and clinical perspective. Biol. Psychiatry 58, 597-604.

Fecteau, S., Knoch, D., Fregni, F., Sultani, N., Boggio, P., and Pascual-Leone, A. (2007a). Diminishing risk-taking behavior by modulating activity in the prefrontal cortex: a direct current stimulation study. J. Neurosci. 27, 12500-12505.

Fecteau, S., Pascual-Leone, A., Zald, D. H., Liguori, P., Theoret, H., Boggio, P. S., and Fregni, F. (2007b). Activation of prefrontal cortex by transcranial direct current stimulation reduces appetite for risk during ambiguous decision making. $J$. Neurosci. 27, 6212-6218.

Feurra, M., Bianco, G., Santarnecchi, E., Del Testa, M., Rossi, A., and Rossi, S. (2011). Frequencydependent tuning of the human motor system induced by transcranial oscillatory potentials. $\mathrm{J}$. Neurosci. 31, 12165-12170. Self-control depletion leads to
Demaree, H. A., Dedonno, M. A., Burns,

Freeman, N., and Muraven, M. (2010).

and pathological gamblers who show deficits in this kind of behavior.

\section{ACKNOWLEDGMENTS}

This study was supported by an ERC starting grant (Inspire 200512), and an ISF (grant 100/10) awarded to Michal Lavidor. The authors would like to thank the reviewers for their helpful comments, which have improved the manuscript.

increased risk taking. Soc. Psychol. Personal. Sci. 1, 175-181.

Gandiga, P. C., Hummel, F. C., and Cohen, L. G. (2006). Transcranial DC stimulation (tDCS): a tool for double-blind sham-controlled clinical studies in brain stimulation. Clin. Neurophysiol. 117, 845-850.

Gehring, W. J., and Willoughby, A. R. (2004). "Are all medial frontal negativities created equal? Toward a richer empirical basis for theories of action monitoring," in Errors, Conflicts and the Brain: Current Opinions on Performance Monitoring, eds M. Ullsperger and M. Falkenstein. (Leipzig: Max Planck Institute of Cognitive Neuroscience), 14-20.

Gianotti, L. R. R., Knoch, D., Faber, P. L., Lehmann, D., Pascual-Marqui, R. D., Diezi, C., Schoch, C., Eisenegger, C., and Fehr, E. (2009). Tonic activity level in the right prefrontal cortex predicts individuals' risk taking. Psychol. Sci. 20, 33-38.

Goldstein, A., Revivo, K., Kreitler, M., and Metuki, N. (2010). Unilateral muscle contractions enhance creative thinking. Psychon. Bull. Rev. 17 895-899.

Gray, J. A. (1987). "The neuropsychology of emotion and personality," in Cognitive Neuropsychology, eds S. M. Stahl, S. D. Iverson, and E. C. Goodman (New York: Oxford University Press), 171-190.

Gray, J. A., and McNaughton, N (2000). The Neuropsychology of Anxiety: An Enquiry into the Functions of the Septo-Hippocampal System. New York: Oxford University Press.

Hare, T. A., Camerer, C. F., and Rangel, A. (2009). Self-control in decisionmaking involves modulation of the vmPFC valuation system. Science 324, 646-648.

Hunt, M. K., Hopko, D. R., Bare, R., Lejuez, C. W., and Robinson, E. V. (2005). Construct validity of the Balloon Analog Risk Task (BART) Assessment 12, 416-428.

Jentsch, J. D., Woods, J. A., Groman, S. M., and Seu, E. (2010). Behavioral characteristics and neural mechanisms mediating performance in a rodent version of the Balloon Analog Risk Task. Neuropsychopharmacology 35, 1797-1806.
Kanai, R., Chaieb, L., Antal, A., Walsh, V., and Paulus, W. (2008). Frequencydependent electrical stimulation of the visual cortex. Curr. Biol. 18, 1839-1843.

Kanai, R., Paulus, W., and Walsh, V. (2010). Transcranial alternating current stimulation (tACS) modulates cortical excitability as assessed by TMS-induced phosphene thresholds. Clin. Neurophysiol. 121 1551-1554.

Kerns, J. G., Cohen, J. D., Macdonald, A. W., Cho, R. Y., Stenger, V. A., and Carter, C. S. (2004). Anterior cingulate conflict monitoring and adjustments in control. Science 303, 1023-1026.

Kinsbourne, M. (1970). The cerebral basis of lateral asymmetries in attention. Acta Psychol. (Amst.) 33, 193-201.

Klimesch, W. (1999). EEG alpha and theta oscillations reflect cognitive and memory performance: a review and analysis. Brain Res. Rev. 29, 169-195.

Klimesch, W., Doppelmayr, M., Russegger, H., and Pachinger, T. (1996) Theta band power in the human scalp EEG and the encoding of new information. Neuroreport 7, 1235-1240.

Knoch, D., Gianotti, L. R. R., PascualLeone, A., Treyer, V., Regard, M., Hohmann, M., and Brugger, P. (2006). Disruption of right prefrontal cortex by low-frequency repetitive transcranial magnetic stimulation induces risk-taking behavior. J. Neurosci. 26, 6469-6472.

Krain, A. L., Wilson, A. M., Arbuckle, R., Castellanos, F. X., and Milham, M. P. (2006). Distinct neural mechanisms of risk and ambiguity: a metaanalysis of decision-making. Neuroimage $32,477-484$

Lee, D., and Seo, H. (2007). Mechanisms of reinforcement learning and decision making in the primate dorsolateral prefrontal cortex. Ann. N. Y. Acad. Sci. 1104, 108-122.

Lejuez, C. W., Aklin, W. M., Bornovalova, M. A., and Moolchan, E. T. (2005). Differences in risk-taking propensity across inner-city adolescent ever-and never-smokers. Nicotine Tob. Res. 7, 71-79. 
Lejuez, C. W., Aklin, W. M., Jones, H. A., Richards, J. B., Strong, D. R., Kahler, C. W., and Read, J. P. (2003a). The Balloon Analogue Risk Task (BART) differentiates smokers and nonsmokers. Exp. Clin. Psychopharmacol. 11, 26-33.

Lejuez, C. W., Aklin, W. M., Zvolensky, M. J., and Pedulla, C. M. (2003b). Evaluation of the Balloon Analogue Risk Task (BART) as a predictor of adolescent real-world risk-taking behaviours. J. Adolesc. 26, 475-479.

Lejuez, C. W., Read, J. P., Kahler, C. W., Richards, J. B., Ramsey, S. E., Stuart, G. L., Strong, D. R., and Brown, R. A. (2002). Evaluation of a behavioral measure of risk taking: the Balloon Analogue Risk Task (BART). J. Exp. Psychol. Appl. 8, 75-84.

Lejuez, C. W., Simmons, B. L., Aklin, W. M., Daughters, S. B., and Dvir, S. (2004). Risk-taking propensity and risky sexual behavior of individuals in residential substance use treatment. Addict. Behav. 29, 1643-1647.

Luu, P., and Tucker, D. M. (2001). Regulating action: alternating activation of midline frontal and motor cortical networks. Clin. Neurophysiol. 112, 1295-1306.

Luu, P., Tucker, D. M., Derryberry, D., Reed, M., and Poulsen, C. (2003). Electrophysiological responses to errors and feedback in the process of action regulation. Psychol. Sci. 14, 47-53.

Luu, P., Tucker, D. M., and Makeig, S. (2004). Frontal midline theta and the error-related negativity: neurophysiological mechanisms of action regulation. Clin. Neurophysiol. 115, 1821-1835.

Marco-Pallares, J., Cucurell, D., Cunillera, T., García, R., AndrésPueyo, A., Münte, T. F., and Rodríguez-Fornells, A. (2008). Human oscillatory activity associated to reward processing in a gambling task. Neuropsychologia 46, 241-248.

Marshall, L., Helgadóttir, H., Mölle, M., and Born, J. (2006). Boosting slow oscillations during sleep potentiates memory. Nature 444, 610-613.

McClure, S. M., Laibson, D. I., Loewenstein, G., and Cohen, J. D. (2004). Separate neural systems value immediate and delayed monetary rewards. Science 306, 503-507.

Miller, E. K., and Cohen, J. D. (2001). An integrative theory of prefrontal cortex function. Annu. Rev. Neurosci. $24,167-202$.

Milner, A. D., Brechmann, M., and Pagliarini, L. (1992). To halve and to halve not: an analysis of line bisection judgements in normal subjects. Neuropsychologia 30, 515-526.

Mohr, P. N. C., Biele, G., and Heekeren, H. R. (2010). Neural processing of risk. J. Neurosci. 30, 6613-6619.

Nash, K., Mcgregor, I., and Inzlicht, M. (2010). Line bisection as a neural marker of approach motivation. Psychophysiology 47, 979-983.

Oldfield, R. C. (1971). The assessment and analysis of handedness: the Edinburgh inventory. Neuropsychologia 9, 97-113.

Paulus, M. P., Hozack, N., Zauscher, B., Mcdowell, J. E., Frank, L., Brown, G. G., and Braff, D. L. (2001). Prefrontal, parietal, and temporal cortex networks underlie decision-making in the presence of uncertainty. Neuroimage $13,91-100$.

Paulus, W. (2011). On the difficulties to separate retinal from cortical origin of phosphenes when using transcranial alternating current stimulation. Clin. Neurophysiol. 121, 987-991.

Pickering, A. D., and Gray, J. A. (1999). "The neuroscience of personality," in Handbook of Personality: Theory and Research, 2nd Edn, eds L. A. Pervin and O. P. John (New York: Guilford Press), 277-299.

Pogosyan, A., Gaynor, L. D., Eusebio, A., and Brown, P. (2009). Boosting cortical activity at beta-band frequencies slows movement in humans. Curr. Biol. 19, 1637-1641.

Rahman, S., Sahakian, B. J., Cardinal, R. N., Rogers, R. D., and Robbins, T. W. (2001). Decision making and neuropsychiatry. Trends Cogn. Sci. 5, 271-277.

Rao, H., Korczykowski, M., Pluta, J., Hoang, A., and Detre, J. A. (2008). Neural correlates of voluntary and involuntary risk taking in the human brain: an fMRI study of the Balloon Analog Risk Task (BART). Neuroimage 42, 902-910.

Ridderinkhof, K. R., Ullsperger, M., Crone, E. A., and Nieuwenhuis, S. (2004). The role of the medial frontal cortex in cognitive control. Science 306, 443-447.

Rogers, R. D., Owen, A. M., Middleton, H. C., Williams, E. J., Pickard, J. D., Sahakian, B. J., and Robbins, T. W. (1999). Choosing between small, likely rewards and large, unlikely rewards activates inferior and orbital prefrontal cortex. J. Neurosci. 19, 9029-9038.

Rosanova, M., Casali, A., Bellina, V. Resta, F., Mariotti, M., and Massimini, M. (2009). Natural frequencies of human corticothalamic circuits. J. Neurosci. 29, 7679-7685.

Sanfey, A. G., Hastie, R., Colvin, M. K., and Grafman, J. (2003a). Phineas gauged: decision-making and the human prefrontal cortex. Neuropsychologia 41, 1218-1229.

Sanfey, A. G., Rilling, J. K., Aronson, J. A., Nystrom, L. E., and Cohen, J. D. (2003b). The neural basis of economic decision-making in the ultimatum game. Science 300 , 1755-1758.

Schonberg, T., Fox, C. R., and Poldrack, R. A. (2011). Mind the gap: bridging economic and naturalistic risktaking with cognitive neuroscience. Trends Cogn. Sci.15, 11-19.

Schutter, D. J. L. G., and Hortensius, R. (2011). Brain oscillations and frequency-dependent modulation of cortical excitability. Brain Stimul. 4, 97-103.

Slovic, P. (1966). Risk-taking in children: age and sex differences. Child Dev. 169-176.

Thut, G., and Miniussi, C. (2009). New insights into rhythmic brain activity from TMS-EEG studies. Trends Cogn. Sci.13, 182-189.

Tranel, D., Bechara, A., and Denburg, N. L. (2002). Asymmetric functional roles of right and left ventromedial prefrontal cortices in social conduct, decision-making, and emotional processing. Cortex $38,589-612$.

Tranel, D., Damasio, H., Denburg, N. L., and Bechara, A. (2005). Does gender play a role in functional asymmetry of ventromedial prefrontal cortex? Brain 128, 2872-2881.

Trepel, C., Fox, C. R., and Poldrack, R. A. (2005). Prospect theory on the brain? Toward a cognitive neuroscience of decision under risk. Brain Res. Cogn. Brain Res. 23 , 34-50.

Vallar, G., and Perani, D. (1986). The anatomy of unilateral neglect after right-hemisphere stroke lesions. A clinical/CT-scan correlation study in man. Neuropsychologia 24 609-622.

van de Vijver, I., Ridderinkhof, K. R., and Cohen, M. X. (2011). Frontal oscillatory dynamics predict feedback learning and action adjustment. J. Cogn. Neurosci. 23 , 4106-4121.

van Ravenzwaaij, D., Dutilh, G., and Wagenmakers, E. J. (2011) Cognitive model decomposition of the BART: assessment and application. J. Math. Psychol. 55, 94-105.

van't Wout, M., Kahn, R. S., Sanfey, A. G., and Aleman, A. (2005). Repetitive transcranial magnetic stimulation over the right dorsolateral prefrontal cortex affects strategic decision-making. Neuroreport 16, 1849-1852.

Wallsten, T. S., Pleskac, T. J., and Lejuez, C. W. (2005). Modeling behavior in a clinically diagnostic sequential risk-taking task. Psychol. Rev. 112, 862.

Zaehle, T., Rach, S., and Herrmann, C. S. (2010). Transcranial alternating current stimulation enhances individual alpha activity in human EEG. PLOS ONE 5, e13766. doi:10.1371/journal.pone.0013766

Zaghi, S., Acar, M., Hultgren, B., Boggio, P. S., and Fregni, F. (2010). Noninvasive brain stimulation with lowintensity electrical currents: putative mechanisms of action for direct and alternating current stimulation. Neuroscientist 16, 285-307.

Conflict of Interest Statement: The authors declare that the research was conducted in the absence of any commercial or financial relationships that could be construed as a potential conflict of interest.

Received: 14 November 2011; paper pending published: 19 December 2011; accepted: 29 January 2012; published online: 14 February 2012.

Citation: Sela T, Kilim A and Lavidor $M$ (2012) Transcranial alternating current stimulation increases risktaking behavior in the Balloon Analog Risk Task. Front. Neurosci. 6:22. doi: 10.3389/fnins.2012.00022

This article was submitted to Frontiers in Decision Neuroscience, a specialty of Frontiers in Neuroscience.

Copyright $(2012$ Sela, Kilim and Lavidor. This is an open-access article distributed under the terms of the Creative Commons Attribution Non Commercial License, which permits noncommercial use, distribution, and reproduction in other forums, provided the original authors and source are credited. 